\title{
Resolvent-based jet noise models: a projection approach
}

\author{
Ethan Pickering, Tim Colonius ${ }^{\dagger}$ \\ California Institute of Technology, Pasadena, CA, USA \\ Peter Jordan $\ddagger$ \\ Institut Pprime, CNRS / Université de Poitiers /ENSMA, 86962 Futuroscope Chasseneuil, France \\ Aaron Towne ${ }^{\S}$ \\ University of Michigan, Ann Arbor, MI, USA
}

\begin{abstract}
Linear resolvent analysis has demonstrated encouraging results for modeling coherent structures in jets when compared against their data-deduced counterparts from highfidelity large-eddy simulations (LES). However, leveraging resolvent modes for reconstructing statistics of the far acoustic field remains elusive. In this study, we use a LES database to produce an ensemble of realizations for the acoustic field that we project on to a limited set of $n$ resolvent modes. The projections are done on a restricted acoustic output domain, $r / D=[5,6]$, and allow for the LES realizations to be recast in the resolvent basis via a datadeduced, low-rank, $n \times n$ cross-spectral density matrix. We find substantial improvements to the acoustic field reconstructions with the addition of a RANS-derived eddy-viscosity model to the resolvent operator. The reconstructions quantitatively match the most energetic regions of the acoustic field across Strouhal numbers, $S t=[0-1]$, and azimuthal wavenumbers, $m=[0,2]$, using only three resolvent modes. Finally, the characteristics of the resulting $n \times n$ covariance matrices are examined and suggest off-diagonal terms may be neglected for $n \leq 3$. Results are presented for round, isothermal, Mach 1.5 and 0.9 jets.
\end{abstract}

\section{Introduction}

This manuscript aims to further work on the development of accurate, reduced-order models for turbulent jet-noise prediction, control, and ultimately, reduction. Our goal is to develop a jet-noise model founded upon the underlying physics of turbulent flows that is low-rank and that provides direct insight into the mechanisms primarily responsible for noise generation. The resolvent analysis, ${ }^{1-4}$ also known as input-output analysis, provides a useful framework for achieving these goals.

The central idea of using the resolvent framework for jet noise modeling is similar to the acoustic analogy ${ }^{5,6}$ whereby a forcing term, related to the statistics of the hydrodynamic near-field turbulence, gives rise, through a linear operator, to the observed far-field sound. The resolvent framework differs in two important ways. First, the operator is decomposed into its singular components that represent the maximal amplification between the forcing and the output. This permits the resulting acoustic field to be described at low rank, and thus limiting the forcing statistics that must be modeled. Secondly, the full linearized Navier-Stokes equations are used as the propagator, and we seek a modal basis that represents both near and far-field coherent structures.

Before recent advances in computational power, the idea of modeling both the hydrodynamic component along with the acoustics would be seen as both unnecessary and computationally taxing. However, the ability to resolve both components of the flow is in fact a benefit. Starting with the experimental findings of Mollo-Christensen ${ }^{7}$ in 1967 and Crow \& Champagne ${ }^{8}$ in 1971, it has become clear that coherent structures

*PhD Student, AIAA Student Member, pickering@caltech.edu

$\dagger$ Frank and Ora Lee Marble Professor of Mechanical Engineering, AIAA Associate Fellow

$\ddagger$ Research Scientist

$\S$ Assistant Professor of Mechanical Engineering, AIAA Member 
in the hydrodynamic near-field are directly responsible for far-field sound. ${ }^{9}$ These structures take the spatiotemporal form of wavepackets and have been found to be the dominant source for aft angle sound, ${ }^{9}$ as well as partial contributors to sideline noise. ${ }^{10,11}$ These wavepackets may be linked to the early works of Crighton \& Gaster ${ }^{12}$ in 1976 (and Michalke ${ }^{13}$ in 1971) who hypothesized that coherent structures could be described as linear instability modes of the mean flow via modal analysis. However, it has now become apparent that the correct representation of wavepackets is that of a highly-amplified response to turbulent fluctuations, which is directly found via the resolvent framework.

Resolvent analysis uses the Singular Value Decomposition (SVD) to decompose the linear terms of the resolvent operator, identifying sets of mutually orthogonal forcing/input and response/output modes, and ranking them in terms of the corresponding energetic gain between the forcing and response. This is particularly important as it allows our model to select the most relevant amplification mechanisms for noise generation. We can then retain a limited set of the resolvent modes to produce a reduced-order model, or in other words, a reduced-rank acoustic analogy.

Several studies have applied resolvent analysis to develop low-rank jet models. ${ }^{3,14,15}$ The existence of relatively low-rank responses in round, turbulent jets was shown by Schmidt et al., ${ }^{4}$ with significant agreement between structures found through spectral proper orthogonal decomposition ${ }^{16}$ (SPOD) of a highfidelity experimentally-verified large-eddy simulations (LES) of jets. ${ }^{17,18}$ Of particular relevance to this study, are "acoustic resolvent modes" induced by performing resolvent analysis with an output domain defined over a region where fluctuations are purely acoustic. Through implementation of an acoustic output domain, resolvent analysis is able to filter out energetic, but acoustically irrelevant structures in the near-field. Jeun et al. ${ }^{3}$ performed such an analysis and found that for a Mach 1.5 jet, at Strouhal number $S t=0.33$ and azimuthal wavenumber $m=0$, that the first resolvent mode reconstructs $57 \%$ of the acoustic energy, but through inclusion of the next 23 resolvent modes the reconstruction improved to $70 \%$ of the acoustic energy. This study looks to perform a similar analysis, in that we compute 20 acoustic resolvent modes and assess how well they reconstruct the acoustic energy. However, we also look to generalize the performance of the resolvent framework across frequencies $S t=0-1$, azimuthal wavenumbers $m=[0-2]$, and for Mach 0.9 and 1.5 jets.

For a resolvent jet model to fully reconstruct flow statistics, and in this case those of the acoustic field, a resolvent-based model must incorporate sub-optimal modes ${ }^{4}$ and correctly describe correlations (i.e. covariance) between modes inherent to turbulent flow. ${ }^{19}$ The latter requirement can be shown via the statistical representation of the resolvent operator ${ }^{16}$

$$
\boldsymbol{S}_{y y}=\boldsymbol{R} \boldsymbol{S}_{f f} \boldsymbol{R}^{*}
$$

where $\boldsymbol{S}_{y y}$ and $\boldsymbol{S}_{f f}$ are the cross-spectral density tensors of the responses and the forcing respectively and $\boldsymbol{R}$ is the resolvent operator. This equation shows that if the forcing CSD, describing spatial correlations or "color" ${ }^{20}$ can be modeled, ${ }^{21}$ then the resolvent operator identically reconstructs the flow statistics, $\boldsymbol{S}_{y y}$. If the forcing were spatially uncorrelated, $\boldsymbol{S}_{f f}=\boldsymbol{\Lambda}$, where $\boldsymbol{\Lambda}$ is a diagonal matrix, then the eigenvectors of $\boldsymbol{S}_{y y}$, which are the SPOD modes of the outputs, are aligned with the eigenvectors of $\boldsymbol{R} \boldsymbol{R}^{*},{ }^{16}$ or the response modes of the resolvent, $\boldsymbol{R}$. Recent work incorporating classical eddy-viscosity models into the resolvent operator showed a significant improvement in the alignment between SPOD and resolvent modes in jets, ${ }^{22}$ reducing the resulting effort required to model the effective $\boldsymbol{S}_{f f}$ by reducing the magnitude of the off-diagonal terms. We utilize the same eddy-viscosity model in the present work.

This paper explores an approach to describe the coupling between resolvent modes that is necessary for reconstructing the acoustic field with a minimal set of resolvent modes. The coupling presents a 'jittering' effect that provides directional and energetic variability in acoustic radiation inherently important for noise prediction. $^{23}$ Determination of the coupling between modes is performed by leveraging an ensemble of LES realizations which are projected on to a limited set (i.e. low-rank) of acoustic resolvent modes. From these projections we attain a (drastically) reduced-order cross-spectral density between the retained modes-a Hermitian, frequency-dependent matrix of size $n \times n$ that accurately represents the acoustic field.

Organization of the manuscript is as follows. We first briefly describe the LES databases used, the main details pertaining to resolvent analysis, and present the statistical description of the resolvent framework for reconstructing the acoustic field and estimating the reduced order covariance matrix in $\S$ II. In $\S$ III we present resolvent modes and LES reconstructions in the resolvent basis for one frequency-wavenumber pair for the Mach 1.5 jet before generalizing the approach to both jets over $S t=[0,1]$ and $m=[0,2]$. In this section we compare the impact of including a RANS eddy viscosity to the resolvent operator and find it 
presents a significantly more efficient resolvent basis. We then conclude by investigating the structure of the 3-mode covariance matrix for $m=0$ for the Mach 1.5 jet as a function of frequency and propose next steps in model development.

\section{Methods}

\section{A. Large Eddy Simulation database}

The LES database and resolvent analysis are described in Schmidt et al. (2018) ${ }^{4}$ and Towne et al. (2018). ${ }^{16}$ For brevity, we summarize the main details here. The LES databases, transonic (Mach 0.9), and supersonic (Mach 1.5) cases, were computed using the flow solver "Charles" and details on numerical methods, meshing, and subgrid-models can be found in Brés et al. (2017, 2018) ${ }^{24,25}$ along with validation cases conducted at PPRIME Institute, Poitiers, France for the Mach 0.9 jet. ${ }^{26}$ The Mach 1.5 round jet corresponds to a Reynolds number $R e_{j}=\rho_{j} U_{j} D / \mu_{j}=1.76 \times 10^{6}$ and for the Mach 0.9 jet $R e_{j}=1.01 \times 10^{6}$, where subscript $j$ gives the value at the center of the jet, $\rho$ is density, $\mu$ is viscosity, and $M_{j}$ is the Mach number $M_{j}=U_{j} / a_{j}$, with $a_{j}$ as the speed of sound at the nozzle centerline.

Throughout the manuscript, variables are non-dimensionalized by the mean jet velocity $U_{j}$, jet diameter $D$, and pressure $\rho_{j} U_{j}^{2}$, with the resulting equation of state $p=\frac{\rho T}{\gamma M_{j}^{2}}$, with $T$ denoting temperature and $\gamma$ the ratio of specific heats. Frequencies are reported in Strouhal number, $S t=f D / U_{j}$, where $f$ is the frequency. The database consists of 10,000 snapshots separated by $\Delta t c_{\infty} / D=0.1$ and 0.2 for $M_{j}=1.5$ and $M_{j}=0.9$ jets, respectively, with $c_{\infty}$ as the ambient speed of sound, and interpolated onto a structured cylindrical grid $x, r, \theta \in[0,30] \times[0,6] \times[0,2 \pi]$, where $x, r, \theta$ are streamwise, radial, and azimuthal coordinates,respectively. Variables are reported by the vector

$$
\boldsymbol{q}=\left[\rho, u_{x}, u_{r}, u_{\theta}, T\right]^{T},
$$

where $u_{x}, u_{r}, u_{\theta}$ are the three velocity components.

For an ensemble approach, the LES database of 10,000 snapshots is segmented into bins of 256 snapshots, with an overlap of $75 \%$, and under the implementation of a Hanning window, resulting in 153 independent realizations of the flow. Each realization is then decomposed in the azimuthal direction and in time. The temporal decomposition provides a resolution of $S t=0.026$ for the $M_{j}=1.5$ jet and $S t=0.0217$ for $M_{j}=0.9$ and the azimuthal decomposition is valid up to $m=68$, however, the acoustically relevant azimuthal wavenumbers are much smaller and only azimuthal wavenumbers $m=[0-2]$ are considered in this paper.

\section{B. Resolvent analysis}

For the round, statistically-stationary, turbulent, jets considered in this manuscript, the compressible NavierStokes, energy, and continuity equations are linearized via a standard Reynolds decomposition, and Fourier transformed both in time and azimuthally to the compact expression

$$
\left(i \omega \mathbf{I}-\mathbf{A}_{m}\right) \boldsymbol{q}_{m, \omega}=\boldsymbol{L}_{m, \omega} \boldsymbol{q}_{m, \omega}=\boldsymbol{f}_{m, \omega},
$$

where $\boldsymbol{L}_{m, \omega}$ is the forward linear operator, $\boldsymbol{f}$ constitutes the nonlinear forcing in each variable, $\omega$ is the frequency, and $m$ is the azimuthal wavenumber. For the purpose of suggesting resolvent analysis as a predictive modeling tool, mean flow quantities used in the operator are derived from a RANS model, fitted closely to the LES mean flow. Although the mean flows are similar, the computation of a RANS model, using the standard $\kappa-\epsilon$ closure equations, also provides an eddy-viscosity field. With previous results of Pickering et al. showing drastically improved agreement between SPOD and resolvent modes with the inclusion of an eddy-viscosity model, we incorporate the eddy-viscosity field from the RANS as $\mu_{T}=c C_{\mu} k^{2} / \epsilon$, where $c$ and $C_{\mu}$ are scaling constants $\left(c=0.2, C_{\mu}=0.0554\right.$ for $M_{j}=1.5$ and $C_{\mu}=0.0623$ for the $M_{j}=0.9$ jet $), k$ is the turbulent kinetic energy field, and $\epsilon$ is the turbulent dissipation field.

Rewriting equation 3 by moving $\boldsymbol{L}_{m, \omega}$ to the right-hand side gives,

$$
\boldsymbol{q}_{m, \omega}=\boldsymbol{L}_{m, \omega}^{-1} \boldsymbol{f}_{m, \omega} .
$$

To specify particular domains for both the response and forcing we may write the above as

$$
\boldsymbol{q}_{m, \omega}=\boldsymbol{L}_{m, \omega}^{-1} \boldsymbol{B} \boldsymbol{f}_{m, \omega}
$$


and define the output variable

$$
\boldsymbol{y}_{m, \omega}=\boldsymbol{C} \boldsymbol{q}_{m, \omega} .
$$

Introducing the compressible energy norm, ${ }^{27}$

$$
\left\langle\boldsymbol{q}_{1}, \boldsymbol{q}_{2}\right\rangle_{E}=\iiint \boldsymbol{q}_{1}^{*} \operatorname{diag}\left(\frac{\bar{T}}{\gamma \bar{\rho} M^{2}}, \bar{\rho}, \bar{\rho}, \bar{\rho}, \frac{\bar{\rho}}{\gamma(\gamma-1) \bar{T} M^{2}}\right) \boldsymbol{q}_{2} r \mathrm{~d} x \mathrm{~d} r \mathrm{~d} \theta=\boldsymbol{q}_{1}^{*} \boldsymbol{W} \boldsymbol{q}_{2},
$$

(where superscript $*$ denotes the complex transpose) via the matrix $\boldsymbol{W}$ to the forcing and response $\left(\boldsymbol{W}_{f}=\right.$ $\left.\boldsymbol{W}_{y}=\boldsymbol{W}\right)$ gives the weighted resolvent operator, $\hat{\boldsymbol{R}}_{m, \omega}$,

$$
\hat{\boldsymbol{R}}_{m, \omega}=\boldsymbol{W}_{y}^{1 / 2} \boldsymbol{C} \boldsymbol{L}_{m, \omega}^{-1} \boldsymbol{B} \boldsymbol{W}_{f}^{-1 / 2} .
$$

For the analysis of the acoustics, the output matrix $C$ is chosen to only include pressure, $p^{\prime}=\frac{\rho^{\prime} \bar{T}+\bar{\rho} T^{\prime}}{\gamma M_{j}^{2}}$, from $x / D=[0,30]$, and $r / D=[5,20]$. Ideally, the LES domain would extend from $r / D=[5,20]$ so that the LES could be directly projected onto the resolvent basis, however, the LES database (i.e. the saved data from the LES) only extends to $r / D=6$. By truncating the resolvent output modes to $r / D=[5,6]$ (after computing the resolvent SVD) and implementing a Moore-Penrose inverse a least square fit of the LES in the resolvent basis can be performed. Additionally, the input matrix, $\boldsymbol{B}$, is chosen to be identity for the most general results, where other studies ${ }^{21}$ suggest the use of a filter based on the location and magnitude of turbulent kinetic energy in the jet. Resolvent modes are then found by taking the singular value decomposition of the weighted resolvent operator giving

$$
\hat{\boldsymbol{R}}_{m, \omega}=\hat{\boldsymbol{U}}_{m, \omega} \boldsymbol{\Sigma} \hat{\boldsymbol{V}}_{m, \omega}^{*}
$$

where the optimal response and forcing modes are contained in the columns of $\boldsymbol{U}_{m, \omega}=\boldsymbol{W}_{y}^{-1 / 2} \hat{\boldsymbol{U}}_{m, \omega}$, with $\boldsymbol{U}_{m, \omega}=\left[\boldsymbol{u}_{m, \omega}^{1}, \boldsymbol{u}_{m, \omega}^{2}, \ldots, \boldsymbol{u}_{m, \omega}^{N}\right], \boldsymbol{V}_{m, \omega}=\boldsymbol{W}_{f}^{-1 / 2} \hat{\boldsymbol{V}}_{m, \omega}, \boldsymbol{V}_{m, \omega}=\left[\boldsymbol{v}_{m, \omega}^{1}, \boldsymbol{v}_{m, \omega}^{2}, \ldots, \boldsymbol{v}_{m, \omega}^{N}\right]$, and $\boldsymbol{\Sigma}=\left(\sigma_{1}, \sigma_{2}, \ldots, \sigma_{N}\right)$ are the optimal gains.

\section{Statistics}

The statistics we are interested in are contained within the cross-spectral density (CSD) tensor, which may be found for the desired output space by multiplying the resolvent equation by its complex conjugate and taking the expectation ${ }^{16}$

$$
\left\langle\boldsymbol{y} \boldsymbol{y}^{*}\right\rangle=\left\langle\hat{\boldsymbol{R}} \boldsymbol{f} \boldsymbol{f}^{*} \hat{\boldsymbol{R}}^{*}\right\rangle
$$

giving

$$
\boldsymbol{S}_{y y}=\hat{\boldsymbol{R}} \boldsymbol{S}_{f f} \hat{\boldsymbol{R}}^{*}
$$

where $\boldsymbol{S}_{y y}$ and $\boldsymbol{S}_{f f}$ are the CSD tensors of the response and the forcing respectively.

As mentioned earlier, this representation shows that if the forcing CSD tensor is known, then the resolvent operator reconstructs the response statistics. However, the forcing CSD is generally unknown. There are at least two potential avenues for modeling this. The first is to directly model $\boldsymbol{S}_{f f}$. To aid in such modeling efforts, $\boldsymbol{S}_{f f}$ may be computed directly from full LES data, or estimated from limited flow statistics. ${ }^{19}$ A second approach is to modify the resolvent operator by supplementing the governing linearized equations with an appropriately linearized turbulence model. In Pickering et al., ${ }^{22}$ an eddy-viscosity model was considered and LES data was used to determine an optimal eddy viscosity field that would align, insofar as possible, the modes of $\boldsymbol{S}_{y y}$ with those of $\boldsymbol{R} \boldsymbol{R}^{*}$. This reduces the magnitude of the off-diagonal terms of $\boldsymbol{S}_{f f}$ that must be modeled. The results showed substantial reductions in these off-diagonal terms, in so far as the near-field coherent structures were concerned.

In this study, we look further into estimating a low-order approximation of the forcing CSD, utilizing the eddy-viscosity approximation of Pickering et al. ${ }^{22}$ but focusing now on the acoustic field. To do so, we return to equation 11 and expand the resolvent operator through its singular value decomposition:

$$
\boldsymbol{S}_{y y}=\hat{\boldsymbol{U}} \boldsymbol{\Sigma} \hat{\boldsymbol{V}}^{*} \boldsymbol{S}_{f f} \hat{\boldsymbol{V}} \boldsymbol{\Sigma} \hat{\boldsymbol{U}}^{*}
$$

and define a covariance matrix, $\mathcal{B}$, as $\mathcal{B}=\boldsymbol{\Sigma} \boldsymbol{V}^{*} \boldsymbol{W}_{f} \boldsymbol{S}_{f f} \boldsymbol{W}_{f} \boldsymbol{V} \boldsymbol{\Sigma}$. This gives

$$
\boldsymbol{S}_{y y}=\hat{\boldsymbol{U}} \mathcal{B} \hat{\boldsymbol{U}}^{*}
$$


which can be rearranged to solve for the covariance matrix,

$$
\mathcal{B}=\boldsymbol{U}^{*} \boldsymbol{W}_{y} \boldsymbol{S}_{y y} \boldsymbol{W}_{y} \boldsymbol{U}
$$

In its current state, the covariance matrix is exact, maintaining a full size of the system and permitting approximately $10^{11}$ degrees of freedom (i.e. $\mathcal{B} \in \mathcal{C}^{5 N_{x} N_{r} \times 5 N_{x} N_{r}}$ ).

To obtain a low-rank model $\mathcal{B}$ from the LES data, which extends only to $r / D=6$, we must modify the above relations. First, we reduce the output space to $x / D=[0,30]$ and $r / D=[5,6]$ in the pressure field and define the associated output matrix as $C_{z}$ where $z$ denotes the new restricted space. Applying $C_{z}$ to both the LES data and resolvent modes gives the ensemble of realizations $\boldsymbol{z}$ and resolvent modes $\boldsymbol{U}_{z}$. In addition to reducing the domain space, we also truncate the resolvent response basis to a limited set of $n$ modes, represented as $\tilde{\boldsymbol{U}}_{z}$. An important consequence of reducing the resolvent domain from $C_{y}$ to $C_{z}$ is a loss of orthogonality. Fortunately, we may still determine a least squares fit of the data by computing the Moore-Penrose inverse of $\boldsymbol{W}_{z}^{1 / 2} \tilde{\boldsymbol{U}}_{z},\left(\boldsymbol{W}_{z}^{1 / 2} \tilde{\boldsymbol{U}}_{z}\right)^{+}=\left(\tilde{\boldsymbol{U}}_{z}^{*} \boldsymbol{W}_{z} \tilde{\boldsymbol{U}}_{z}\right)^{-1} \tilde{\boldsymbol{U}}_{z}^{*} \boldsymbol{W}_{z}^{1 / 2}$, and projecting onto the CSD of $z$ to estimate

$$
\tilde{\mathcal{B}}=\left(\boldsymbol{W}_{z}^{1 / 2} \tilde{\boldsymbol{U}}_{z}\right)^{+*} \boldsymbol{W}_{z}^{1 / 2} \boldsymbol{S}_{z z} \boldsymbol{W}_{z}^{1 / 2}\left(\boldsymbol{W}_{z}^{1 / 2} \tilde{\boldsymbol{U}}_{z}\right)^{+},
$$

where $\left(\boldsymbol{W}_{z}^{1 / 2} \tilde{\boldsymbol{U}}_{z}\right)^{+} \in \mathcal{C}^{N_{z} \times n}$ and the covariance matrix then reduces to size $n \times n$, (i.e. degrees of freedom are $10^{1}$ ). With $\tilde{\mathcal{B}}$, we may ask the following questions: (1) How well does $\tilde{\mathcal{B}}$ reconstruct $\boldsymbol{S}_{z z}$ in the truncated resolvent basis? (2) Can $\tilde{\mathcal{B}}$ reconstruct a more general space (e.g. $\boldsymbol{C}=\boldsymbol{I}$ )? (3) How does a reduced knowledge of $\boldsymbol{S}_{z z}$ affect the estimation of $\tilde{\mathcal{B}}$ (and its implications to the prior two questions)? (4) May $\tilde{\mathcal{B}}$ be further reduced (e.g. neglect off-diagonal terms)? (5) Can $\tilde{\mathcal{B}}$ be modeled?

To address the first question, how well does $\tilde{\mathcal{B}}$ reconstruct $\boldsymbol{S}_{z z}$, the estimated response CSD is computed by

$$
\tilde{\boldsymbol{S}}_{z z}=\tilde{\boldsymbol{U}}_{z} \tilde{\mathcal{B}} \tilde{\boldsymbol{U}}_{z}^{*}
$$

We can then determine the accuracy of the reconstructions by comparing the difference of root mean square (RMS) values in terms of decibels:

$$
\Delta d B=20 \log \left(\sqrt{\frac{1}{k-1} \operatorname{diag}\left(\tilde{\boldsymbol{S}}_{z z}-\boldsymbol{S}_{z z}\right)^{2}}\right),
$$

where $k$ is the number of realizations. Alternatively, an energy norm of the error could be taken, however, analyzing an energy norm when considering acoustics may be misleading due to the range of magnitude in the far-field.

To assess how well a more general space may be reconstructed with $\tilde{\mathcal{B}}$ we use the original forcing modes computed from the resolvent and recalculate the responses with the response restriction matrix set to identity. This is computed as

$$
\tilde{\boldsymbol{U}}_{q}=\boldsymbol{L}^{-1} \tilde{\boldsymbol{V}}_{y}
$$

where $\boldsymbol{U}_{q}$ is the set of response modes of the full domain from the set of forcing modes $\boldsymbol{V}_{y}$ of the $\boldsymbol{C}_{y}$ restricted domain. We can then estimate the full state CSD,

$$
\hat{\boldsymbol{S}}_{q q}=\tilde{\boldsymbol{U}}_{q} \tilde{\mathcal{B}} \tilde{\boldsymbol{U}}_{q}^{*} .
$$

Considering flow statistics may be sparse or limited in practical applications, a natural question arises in whether a reduced $\boldsymbol{S}_{z z}$ can lead to a similar $\tilde{\mathcal{B}}$ found from the full $\boldsymbol{S}_{z z}$ or, at the least, a useful $\tilde{\mathcal{B}}$ for then predicting quantities of $\boldsymbol{S}_{z z}$. We now define a reduced response CSD $\breve{\boldsymbol{S}}_{z z}$, as the diagonal of the response $\mathrm{CSD}$, or the power spectral density (PSD), and a partially-informed $\tilde{\mathcal{B}}$ given by

$$
\tilde{\mathcal{B}}=\left(\boldsymbol{W}_{z}^{1 / 2} \tilde{\boldsymbol{U}}_{z}\right)^{+*} \boldsymbol{W}_{z}^{1 / 2} \breve{S}_{z z} \boldsymbol{W}_{z}^{1 / 2}\left(\boldsymbol{W}_{z}^{1 / 2} \tilde{\boldsymbol{U}}_{z}\right)^{+} \cdot
$$

The estimation is then again

$$
\tilde{\boldsymbol{S}}_{z z}=\tilde{\boldsymbol{U}}_{z} \tilde{\mathcal{B}} \tilde{\boldsymbol{U}}_{z}^{*}
$$

Similarly, estimation of a more general state with reduced statistics can also be performed.

Finally, the structure of the reduced-order covariance matrix $\tilde{\mathcal{B}}$ is examined to determine the feasibility of modeling such a matrix. We may first assess how well the truncated resolvent basis directly captures 

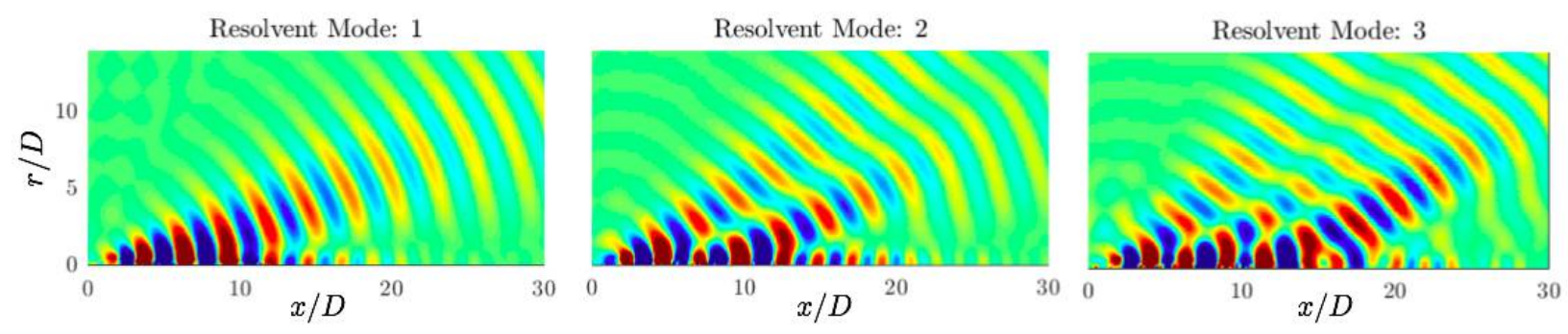

Figure 1. The first three resolvent modes of fluctuating pressure, $q_{p^{\prime}}$. Red and blue contours vary from $\pm 50 \%$ of the maximum fluctuating pressure of each mode, $\pm 0.5\left\|q_{p^{\prime}}\right\|_{\infty} \cdot M_{j}=1.5, S t=0.26, m=0$.

the off-diagonal information of $\boldsymbol{S}_{z z}$ by examining whether $\tilde{\mathcal{B}}$ is diagonally dominant. To make a qualitative assessment of the diagonal dominance we normalize $\tilde{\mathcal{B}}$ by its diagonal components

$$
\tilde{\mathcal{B}}_{\text {norm }}=\operatorname{diag}(\tilde{\mathcal{B}})^{-1 / 2} \tilde{\mathcal{B}} \operatorname{diag}(\tilde{\mathcal{B}})^{-1 / 2}
$$

such that the magnitude of all entries range from 0-1 and the diagonal reads unity, therefore, providing a relative measure of the importance of each diagonal from $0-1$. In the case where $\tilde{\mathcal{B}}_{\text {norm }}=\boldsymbol{I}$, the truncatedresolvent model would present a basis that inherently captures the off-diagonal information of $\boldsymbol{S}_{z z}$ and eliminates the need for further 'coloring' of the resolvent modes to reconstruct $\tilde{\boldsymbol{S}}_{z z}$.

However, modeling modes which perfectly capture the coloring of turbulent flows is difficult. As such, we look closer at the inherent terms of $\tilde{\mathcal{B}}$

$$
\tilde{\mathcal{B}}=\tilde{\boldsymbol{\Sigma}}_{z} \tilde{\boldsymbol{V}}^{*} \boldsymbol{W}_{f} \boldsymbol{S}_{f f} \boldsymbol{W}_{f} \tilde{\boldsymbol{V}} \tilde{\boldsymbol{\Sigma}}_{z}
$$

we see that $\tilde{\boldsymbol{\Sigma}}_{z}$, the corrected resolvent gain from altering the domain to $\boldsymbol{C}_{z}$ found by

$$
\tilde{\boldsymbol{\Sigma}}_{z}=\tilde{\boldsymbol{\Sigma}}_{y} \frac{\tilde{\boldsymbol{U}}_{z}^{*} \boldsymbol{W}_{z} \tilde{\boldsymbol{U}}_{z}}{\tilde{\boldsymbol{U}}_{y}^{*} \boldsymbol{W}_{y} \tilde{\boldsymbol{U}}_{y}}
$$

is directly determined by the resolvent, leaving only $\tilde{\boldsymbol{V}}^{*} \boldsymbol{W}_{f} \boldsymbol{S}_{f f} \boldsymbol{W}_{f} \tilde{\boldsymbol{V}}$ to be modeled. These set of terms may also be viewed as $\tilde{\boldsymbol{V}}^{*} \boldsymbol{W}_{f} \boldsymbol{S}_{f f} \boldsymbol{W}_{f} \tilde{\boldsymbol{V}}=\left(\tilde{\boldsymbol{V}}^{*} \boldsymbol{W}_{f} \boldsymbol{f}\right)\left(\boldsymbol{f}^{*} \boldsymbol{W}_{f} \tilde{\boldsymbol{V}}\right)$ where $\boldsymbol{\beta}=\tilde{\boldsymbol{V}}^{*} \boldsymbol{W}_{f} \boldsymbol{f}$ represents the projection of the LES forcing onto the resolvent forcing basis, giving the effective magnitude, and relative phase, of each resolvent forcing mode for proper reconstruction of the flow. Therefore, we chose to investigate the magnitude and phase components of the complex covariance matrix $\tilde{\mathcal{B}}$ for informing a future noise model.

\section{Results}

\section{A. Resolvent analysis and LES reconstructions}

We begin by providing detailed results for a single frequency and azimuthal wavenumber pair of the $M_{j}=1.5$ jet using the RANS eddy-viscosity resolvent operator. Figure 1 presents the first three resolvent modes computed with a restricted acoustic output domain $(r / D=[5,20]$ in the fluctuating pressure field) and recast in the full domain by $\boldsymbol{U}_{q}=\boldsymbol{L}^{-1} \boldsymbol{V}_{y}$ for $M_{j}=1.5, S t=0.26$, and $m=0$. The associated gain of these modes, normalized by the first resolvent gain are $[1,0.17,0.15]$ (and slowly decreasing with higher modes), indicating the first resolvent mode has at least six times the amplification to its associated forcing than the following resolvent modes.

The resolvent response modes show a particular pattern of acoustic beams. For the first mode there is a single, energetic acoustic beam, propagating at a shallow angle to the jet axis. The first sub-optimal mode consists of two acoustic beams, similar to what was found by Jeun et al. ${ }^{3}$ This pattern continues to the next sub-optimal mode, with three beams located at the perimeter of the first sub-optimal. Although not shown, this behavior continues for further sub-optimal modes.

The resolvent response modes can now be used to determine the covariance matrix $\tilde{\mathcal{B}}$. However, before directly computing $\tilde{\mathcal{B}}$, we first provide an example of individual reconstructions of the LES realizations. To 


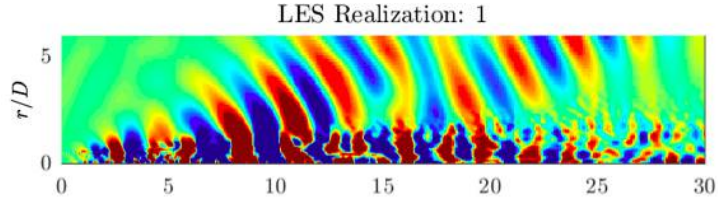

LES Realization: 2
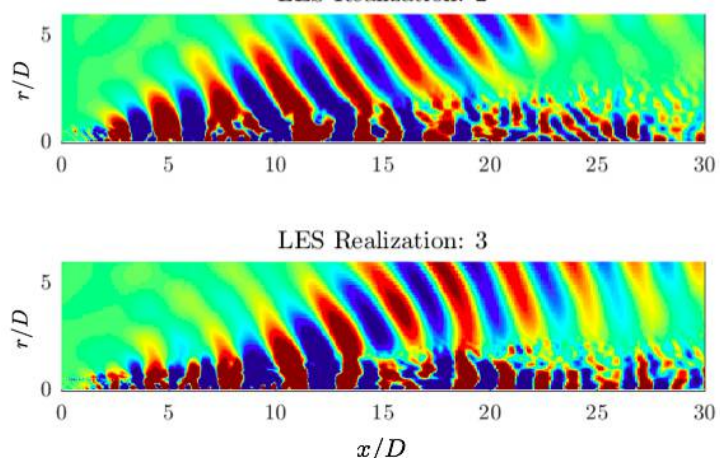

Figure 2. Three realizations of the LES pressure field at $M_{j}=1.5, S t=0.26, m=0$. Red and blue contours vary from $\pm 50 \%$ of the maximum fluctuating pressure of each mode, $\pm 0.5\left\|q_{p^{\prime}}\right\|_{\infty}$.
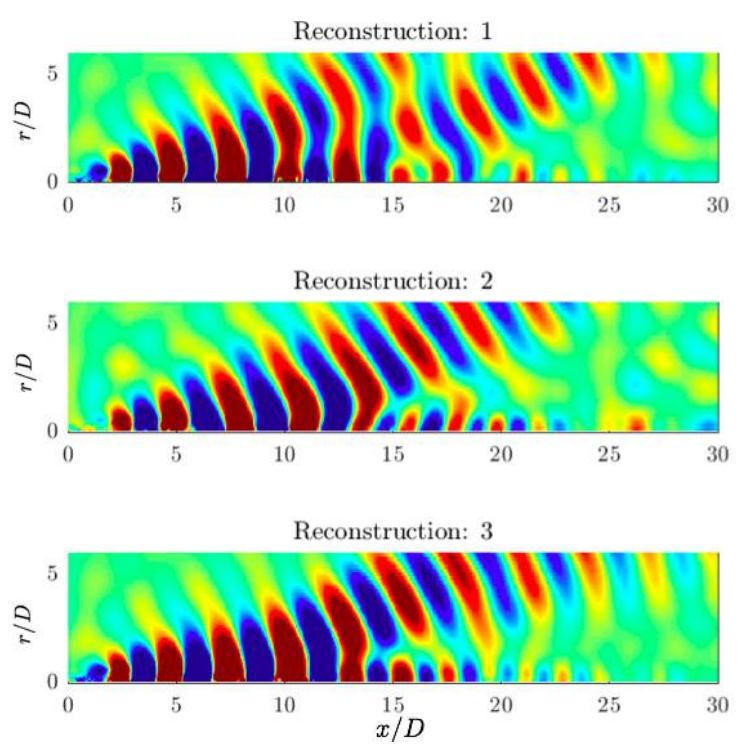

Figure 3. Reconstructions of the corresponding LES realizations using the 3-mode resolvent basis. Contour values are identical to figure $2 . M_{j}=1.5$, $S t=0.26, m=0$.
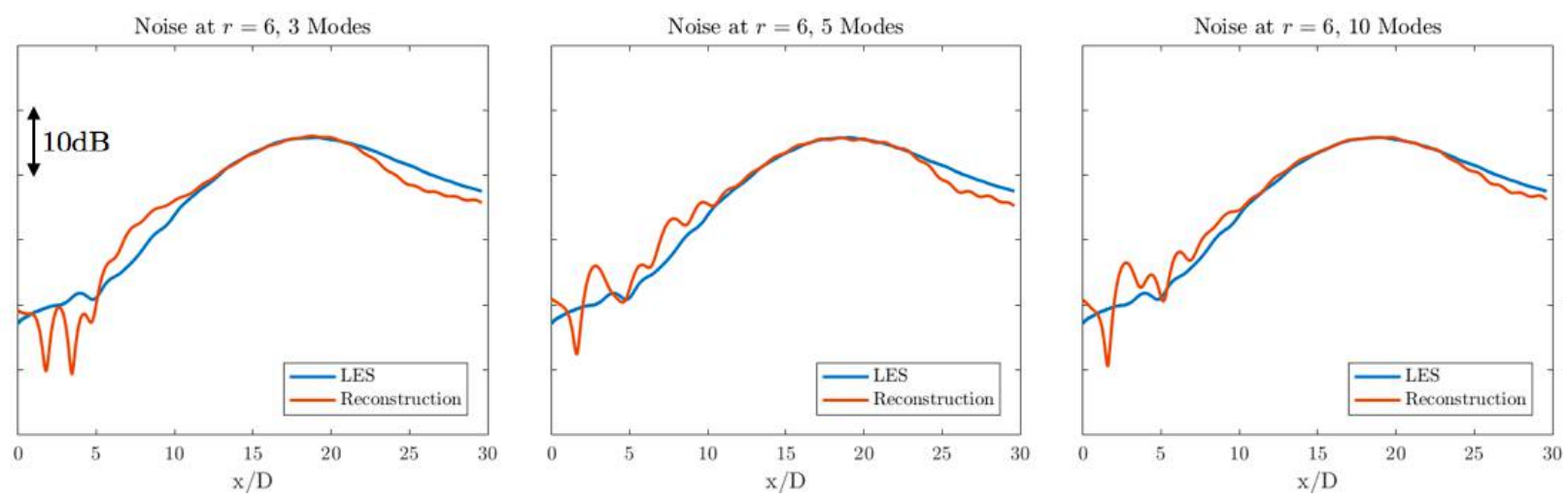

Figure 4. Comparison of pressure RMS values by dB at $r / D=6$ for the LES ensemble and reconstructions in the resolvent basis using 3,5 , and 10 resolvent modes.

do this we return to the equation

$$
\tilde{\mathcal{B}}=\tilde{\boldsymbol{U}}_{z}^{+*} \boldsymbol{W}_{z} \boldsymbol{S}_{z z} \boldsymbol{W}_{z} \tilde{\boldsymbol{U}}_{z}^{+}
$$

and expanding $\boldsymbol{S}_{z z}$ to $\boldsymbol{z z}^{*}$ shows that $\tilde{\mathcal{B}}$ is the covariance of the ensemble of projection coefficients, $\boldsymbol{\alpha}=$ $\tilde{\boldsymbol{U}}_{z}^{+*} \boldsymbol{W}_{z} \boldsymbol{z}$ of the LES data in the resolvent basis. Figure 2 and 3 provide three independent LES realizations and their reconstructions (using $\tilde{\boldsymbol{U}}_{q}$ ) in a 3-mode truncated resolvent basis.

While the resolvent gain is only optimal over the region $r / D=[5-20]$, the three resolvent modes are able to accurately reconstruct the different radiation patterns evident in the realizations. Clearly there is constructive and destructive reinforcement amongst the three resolvent modes in order to produce the LES realizations.

To quantitatively assess how well the resolvent basis reconstructs the LES realizations, we compute and compare the RMS pressure values by $\mathrm{dB}$ at $r / D=6$ in figure 4 , again for $S t=0.26, m=0$ over all $k=153$ realizations. In addition to the 3 resolvent mode basis, results are also shown for 5 and 10 mode bases. With just three modes we see that the peak in directivity is well captured, with minor improvements (and diminishing returns) in the off-peak directivity with increasing numbers of modes. 


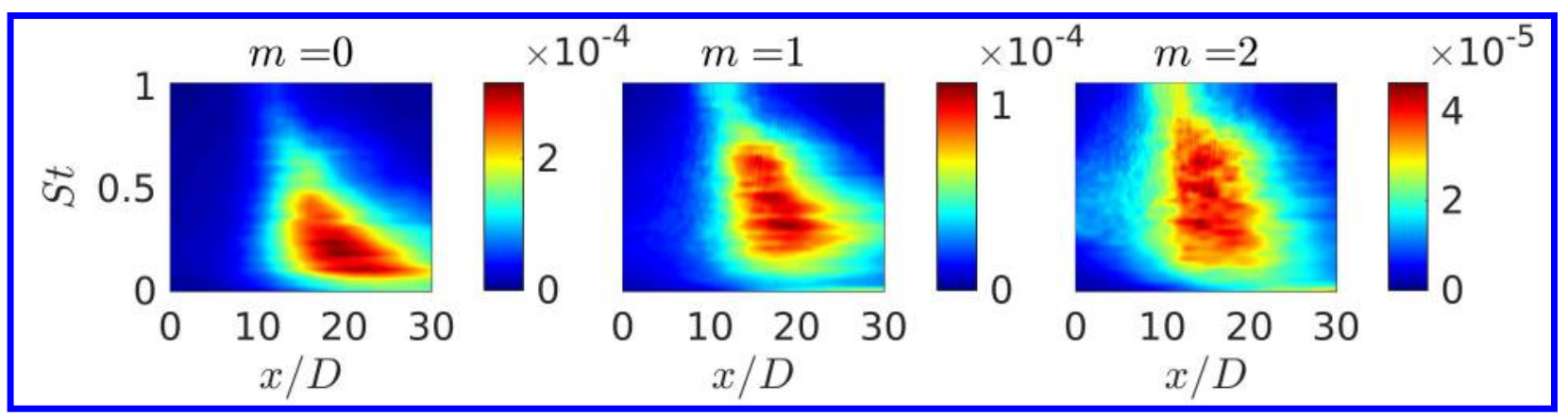

Figure 5. RMS of LES pressure fluctuations of the $M_{j}=1.5$ jet at radial surface $r / D=6$ from $S t=[0,1]$ and $x / D=[0,30]$ for three azimuthal wavenumbers, $m=[0,2]$.

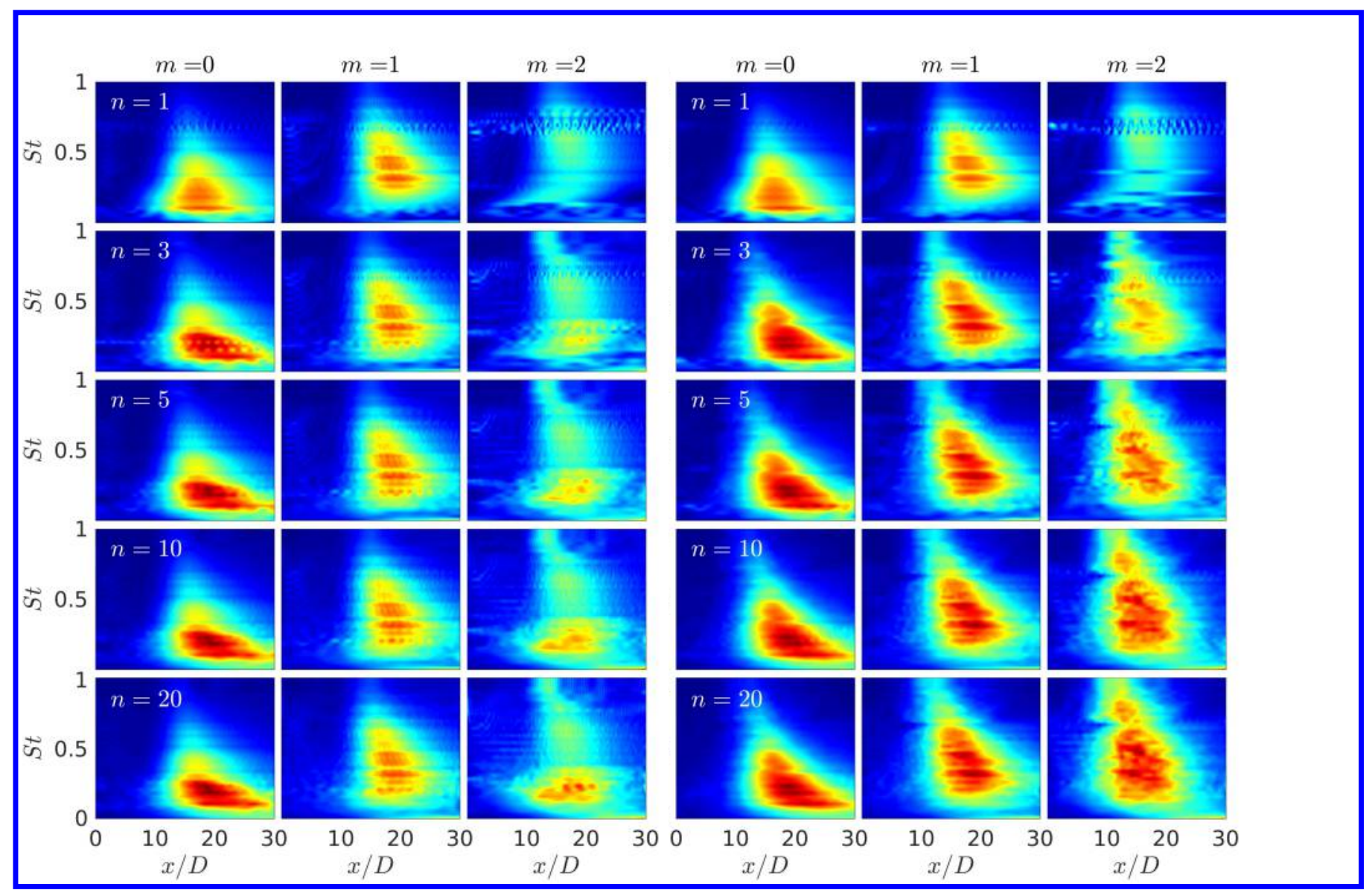

Figure 6. RMS of resolvent reconstructions of the $M_{j}=1.5$ jet, without an eddy-viscosity model (left) and with a RANS eddy-viscosity model (right), at radial surface $r / D=6$ from $S t=[0,1]$ and $x / D=[0,30]$ for three azimuthal wavenumbers, $m=[0,2]$, from left to right and using $n=1,3,5,10,20$ modes from top to bottom. Contours and axes mirror those of figure 5.

\section{B. Eddy viscosity model}

We now expand our analysis to Strouhal numbers ranging from 0 to 1 and azimuthal wavenumbers 0-2 and assess the overall ability of the truncated resolvent basis to reconstruct the acoustic field, as well as determine the utility of implementing an eddy-viscosity model for noise modeling. We continue comparisons of the LES data and resolvent reconstructions by RMS pressure fluctuations at $r / D=6$, which are now plotted over a range of frequencies $(S t=[0,1])$ and azimuthal modes $(m=[0-2])$ in figure 5 . In figure 6 , the resolvent-basis reconstructions of the pressure fluctuations are shown for bases of size 1, 3, 5, 10, and 20. 


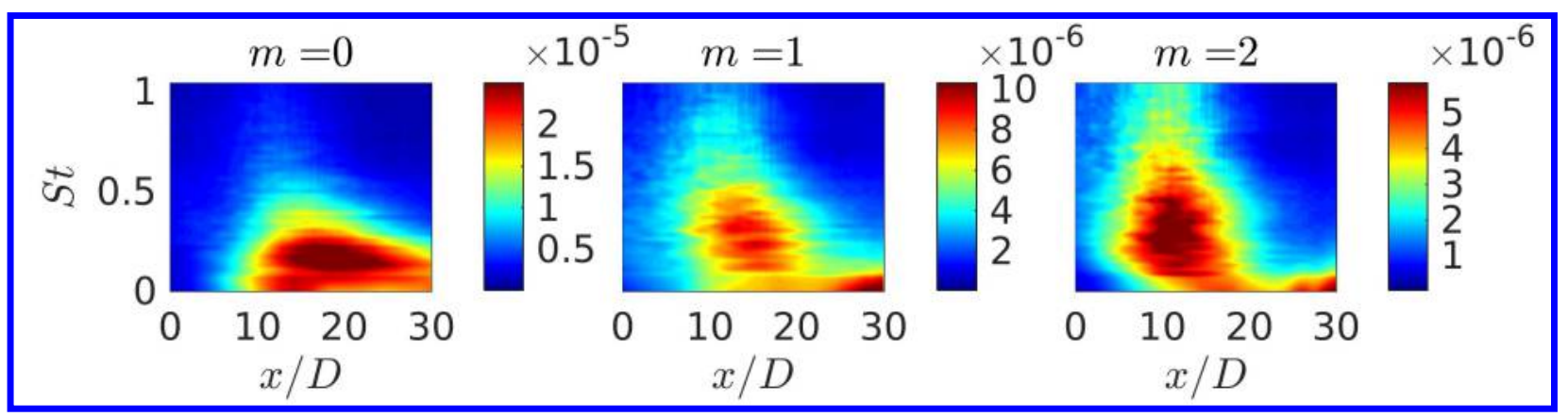

Figure 7. RMS of LES pressure fluctuations of the $M_{j}=0.9$ jet at radial surface $r / D=6$ from $S t=[0,1]$ and $x / D=[0,30]$ for three azimuthal wavenumbers, $m=[0,2]$.

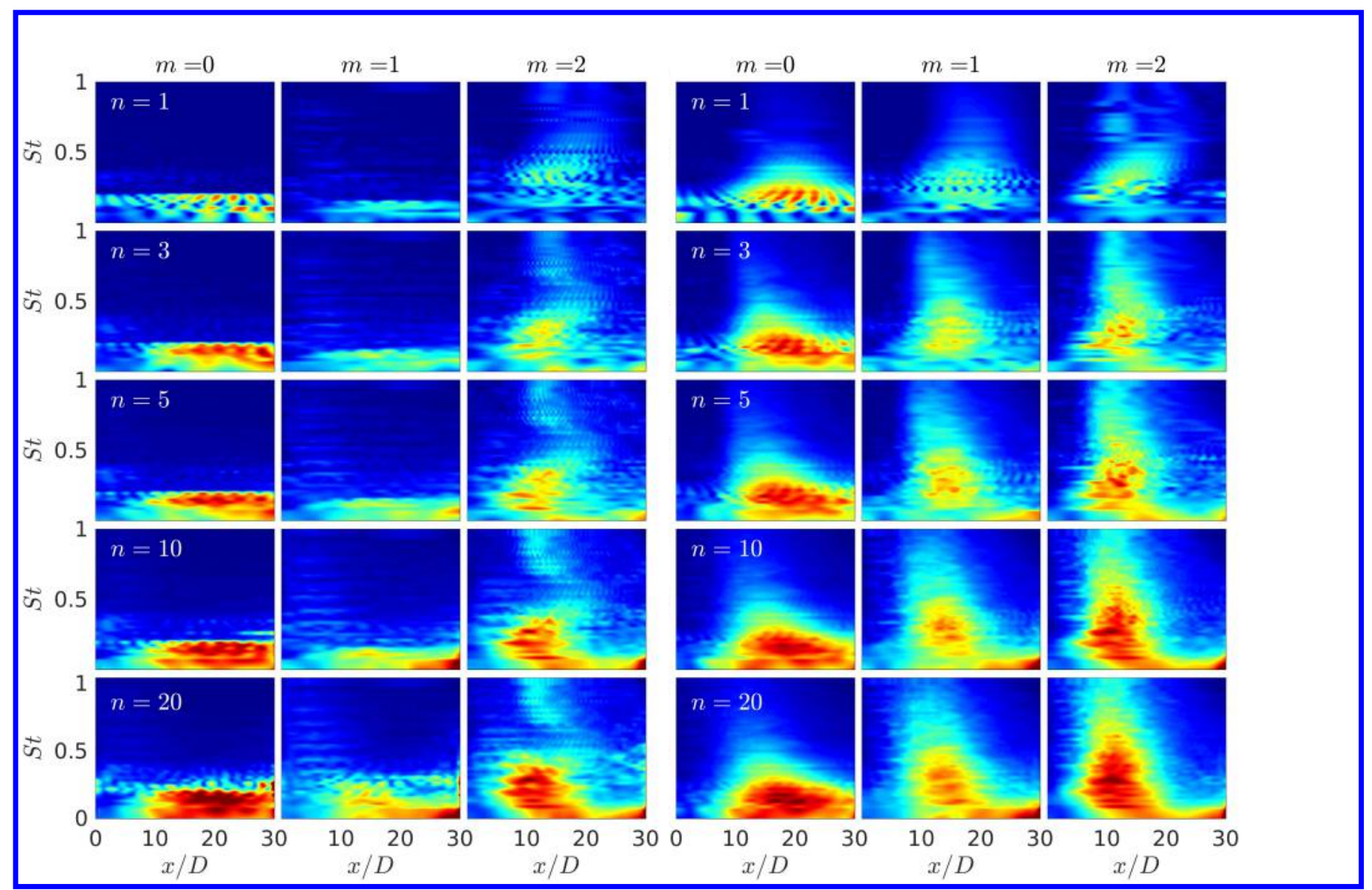

Figure 8. RMS of resolvent reconstructions of the $M_{j}=0.9$ jet, without an eddy-viscosity model (left) and with a RANS eddy-viscosity model (right), at radial surface $r / D=6$ from $S t=[0,1]$ and $x / D=[0,30]$ for three azimuthal wavenumbers, $m=[0,2]$, from left to right and using $n=1,3,5,10,20$ modes from top to bottom. Contours and axes mirror those of figure 7 .

The left and right columns show resolvent bases without and with the eddy viscosity model, respectively. For the computations without eddy viscosity, a constant turbulent Reynolds number $\left(\operatorname{Re}_{T}=3 \times 10^{4}\right)$ is used.

A visual comparison between these columns, as well as figure 9 presented later, shows the clear advantage that the eddy-viscosity model provides, especially when two or more resolvent modes are retained. Interestingly, the rank-1 model appears to be quite similar both with and without eddy viscosity, which is likely due to the Kelvin-Helmholtz mechanism that dominates (i.e. as the leading resolvent mode) much of the frequency-wavenumber space considered, ${ }^{28}$ and which is less sensitive to the eddy-viscosity model than 


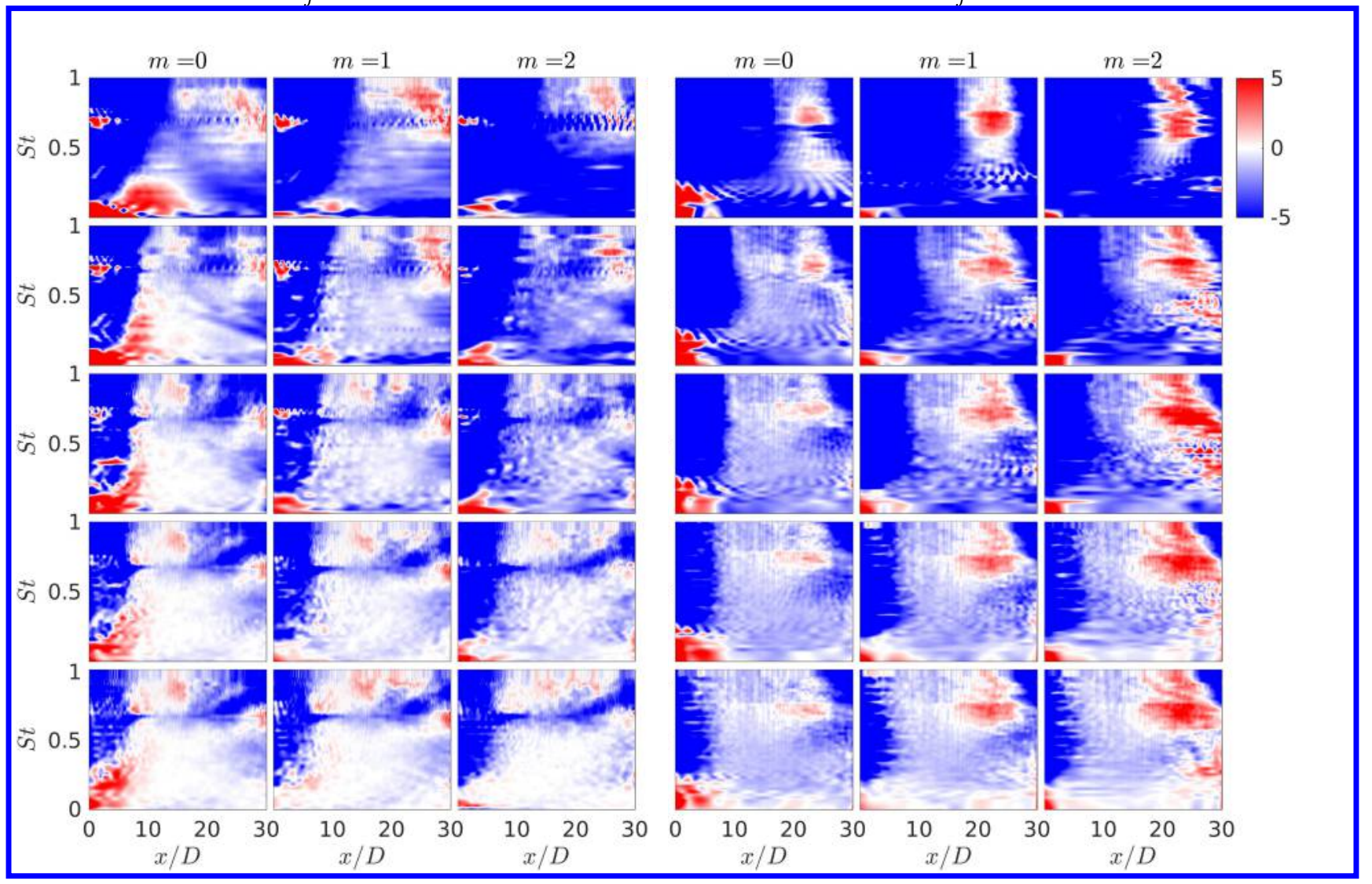

Figure 9. Difference in decibels, $d B$, between the RMS Figure 10. Difference in decibels, $d B$, between the values of LES and the eddy-viscosity resolvent recon- RMS values of LES and the eddy-viscosity resolvent structions for the $M_{j}=1.5$ jet. Axes mirror those of reconstructions for the $M_{j}=0.9$ jet. Axes mirror those figure 5 with contours set at $\pm 5 \mathrm{~dB}$. of figure 7 with contours set at $\pm 5 \mathrm{~dB}$.

the sub-optimal modes. ${ }^{22}$ The rank-1 results are also similar to those of Sinha et al. ${ }^{29}$ who computed a rank-1 model via the parabolized stability equations and projected onto the first SPOD-mode at each $S t-m$ pair. However, we show here that once additional modes are included the reconstructions are substantially improved and the eddy-viscosity model shows superior performance; the 20-mode, eddy-viscosity model is indistinguishable from the LES for all frequencies and azimuthal modes, while even the 3-modes model is quantitatively accurate for $m=0$ and $m=1$.

In figures 7 and 8, we plot the LES and reconstructed pressure fluctuations, respectively, for the $M_{j}=0.9$ jet. We see many similar behaviors to the $M_{j}=1.5$ case, most notably the superiority of the eddy-viscosity resolvent modes, which achieve nearly complete reconstruction of the full LES pressure with just 20 modes. However, it is also apparent that a larger number of modes is required to accurately reconstruct the modes compared to the $M_{j}=1.5$ case. For example, about 10 modes are required to obtain a reasonable quantitative match (compared to just 3 modes at $M_{j}=1.5$ ).

In order to get a clearer view of the accuracy of the reconstructions, we plot in figures 9 and 10 the difference between the LES and reconstructed pressure fields in $\mathrm{dB}$ for both $M_{j}=1.5$ and $M_{j}=0.9$, but concentrating now just on the model with eddy viscosity. Generally, more modes are required for low frequencies, particularly for the axisymmetric mode, and higher azimuthal modes over all frequencies. This is generally consistent with the lack of clear low-rank (Kelvin-Helmholtz) response at those conditions. ${ }^{4}$ Likewise, the region $x / D=[0-10]$ is consistently underpredicted at all frequencies by the resolvent basis. At $r / D=6$, this region generally consists of sideline noise which is more than $20 \mathrm{~dB}$ lower than the peak noise and it is unsurprising that low-rank models based on maximizing the response over $0<x / D<30$ would underpredict regions that are significantly less energetic. 

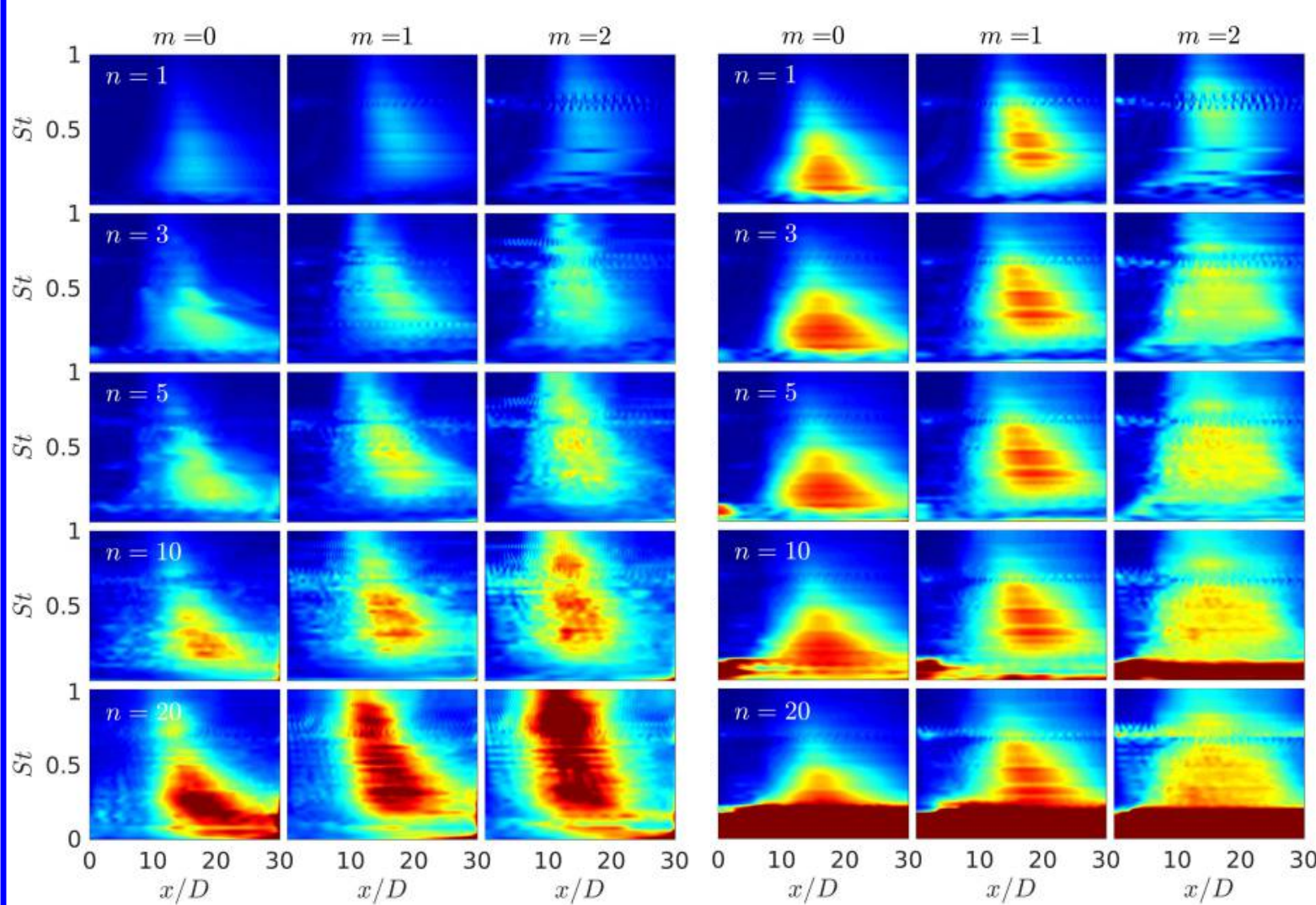

1020
$x / D$

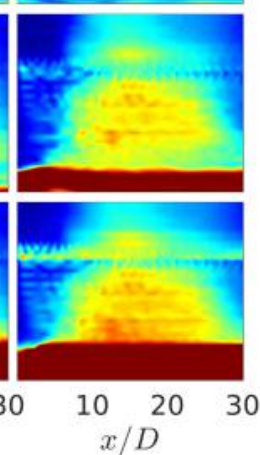

Figure 11. RMS of resolvent reconstructions for the Figure 12. RMS of resolvent reconstructions for the $M_{j}=1.5$ jet using only the PSD of the CSD matrix, $M_{j}=1.5$ jet using only the diagonal components of $\tilde{\mathcal{B}}$ $S_{z z}$, with a RANS eddy-viscosity model, at radial sur- matrix, with a RANS eddy-viscosity model, at radial face $r / D=6$ from $S t=[0,1]$ and $x / D=[0,30]$ for three surface $r / D=6$ from $S t=[0,1]$ and $x / D=[0,30]$ for azimuthal wavenumbers, $m=[0,2]$, from top to bot- three azimuthal wavenumbers, $m=[0,2]$, from top to tom and using $n=1,3,5,10,20$ modes from left to right. bottom and using $n=1,3,5,10,20$ modes from left to Axes mirror those of figure 5, while the contours are right. Axes and contours mirror those of figure 5. reduced by a factor of 17.8 (i.e. $25 \mathrm{~dB}$ ).

\section{Modeling with uncorrelated data}

We may inquire whether the off-diagonal components of the original CSD are important to the estimated $\tilde{\mathcal{B}}$ and the resulting reconstruction of the RMS pressure fluctuations. Figure 11 shows results for the $M_{j}=1.5$ case when only retaining the diagonal components of $\boldsymbol{S}_{z z}$. While the results show qualitatively similar directivity, the resulting magnitude is vastly underpredicted by the reconstructions (note that the contours have been shifted by $25 \mathrm{~dB}$ from the previous figures). These results seem to confirm the importance of "jitter". ${ }^{23,30}$ Only by providing information about the coherence of the data (i.e. off-diagonals of the CSD) can the model determine the correlations, in phase and amplitude, between resolvent modes (for example the one, two, and three-beam), such that through constructive interference, produce the observed radiation.

Alternatively, rather than training $\tilde{\mathcal{B}}$ with only the PSD of the data, we may estimate $\tilde{\mathcal{B}}$ using the full CSD and then assess the importance of the off-diagonal components of $\tilde{\mathcal{B}}$ by only utilizing the diagonal components to reconstruct the LES statistics. Using only the diagonal components of the estimated CSD provides the reconstructions in figure 12 , now using the same contours as those of figure 5 . For a limited set of modes, such as $n=3$, the reconstructions perform rather well, only slightly underpredicting the overall magnitude of the RMS values. However, the addition of more modes, $n>5$, leads to significant errors at low frequencies. The deteriorating reconstruction as $n$ increases is a result of the diagonal dominance of $\tilde{\mathcal{B}}$. If $\tilde{\mathcal{B}}$ is diagonally dominant, then the diagonal reconstruction is valid, if not, then the correlations between modes are crucial for recovering the reconstruction. We investigate this hypothesis in the next section by examining the structure of $\tilde{\mathcal{B}}$. 


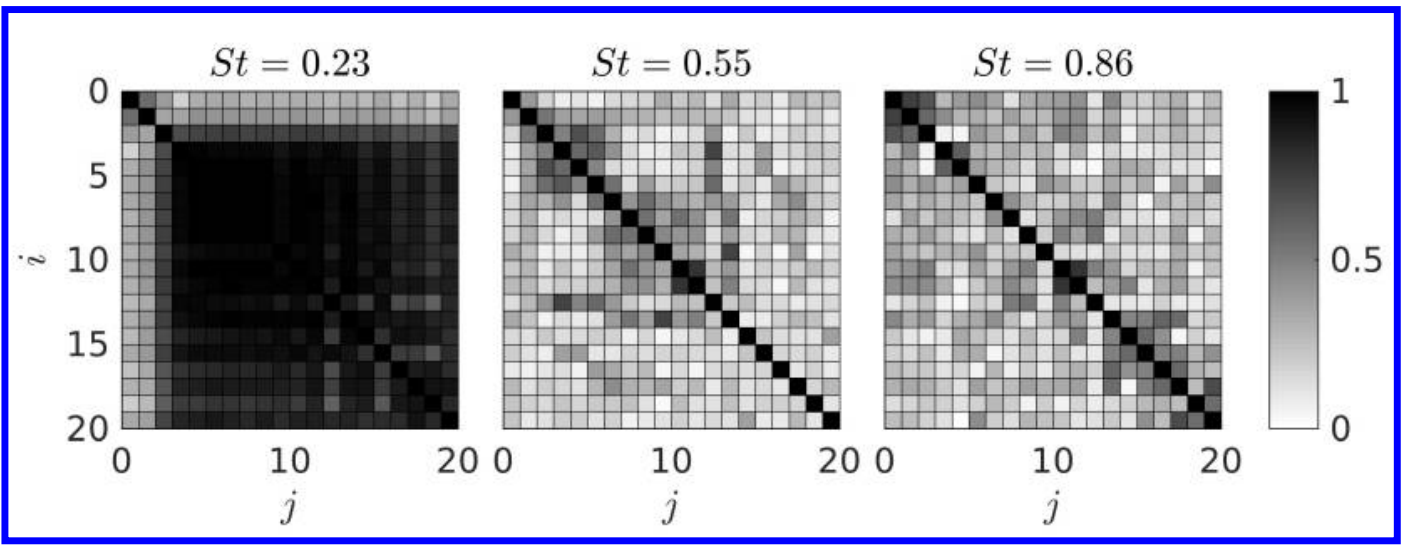

Figure 13. Normalized magnitude of the estimated forcing $\operatorname{CSD}, \tilde{\mathcal{B}}_{\text {norm }}=\operatorname{diag}(\tilde{\mathcal{B}})^{-1 / 2} \tilde{\mathcal{B}} \operatorname{diag}(\tilde{\mathcal{B}})^{-1 / 2}$.

\section{Frequency characteristics of the CSD}

Finally, we investigate characteristics of the estimated CSD matrix $(\tilde{\mathcal{B}})$, using the full CSD data and eddyviscosity model basis, in the hope of identifying trends which may be generalized for a noise model. First, we assess the dominance of the diagonal components in $\tilde{\mathcal{B}}$ through normalizing the matrix by its diagonal components such that the magnitude of all components lie between $0-1$ and all diagonal terms read unity, $\tilde{\mathcal{B}}_{\text {norm }}=\operatorname{diag}(\tilde{\mathcal{B}})^{-1 / 2} \tilde{\mathcal{B}} \operatorname{diag}(\tilde{\mathcal{B}})^{-1 / 2}$. Figure 13 provides the normalized $\tilde{\mathcal{B}}$ for $S t=[0.23,0.55,0.86]$ at $m=0$ showing that the diagonal assumption is particularly poor at lower frequencies, but relatively valid at moderate and high frequencies, explaining the inferior performance at low frequencies in figure 12 . The lack of diagonal dominance at low-frequencies is likely the result of the comparison domain chosen, $r / D=$ $[5-6]$, as near-field behavior extends into this domain has been found to be of significantly higher rank. ${ }^{4,31}$ Additionally, the diagonal dominance is relative to the number of modes present in the truncated basis. If only $n=3$ modes are kept the diagonal assumption is valid for all frequencies, presenting the rather accurate results of row two in 12. This last observation is crucial as we seek a lowest rank model, therefore the use of only $n=3$ modes is preferential for a reduced order model.

Further, the diagonally dominant structure of $\tilde{\mathcal{B}}$ for many of the $S t-m$ pairs provides a sense of the optimality of the current resolvent basis. Even though the off-diagonal components of $S_{z z}$ are necessary for capturing the effect of jitter, the resulting $\tilde{\mathcal{B}}$ off-diagonals may be neglected due to the ability of the resolvent basis to directly capture the statistics of the acoustic field. In other words, the off-diagonal terms of $\boldsymbol{S}_{z z}$ are directly projected onto the diagonals of $\tilde{\mathcal{B}}$. This is due to the resolvent basis inherently possessing characteristics of jitter (i.e. variability in beam location and directivity ${ }^{23}$ ), as the basis provides multiple acoustic beams which vary in axial extent of beams, directivity, and phase.

For the remainder of this section we limit the analysis to the axisymmetric mode, $m=0$, and use the rank-3 model, a $3 \times 3$ complex-valued $\tilde{\mathcal{B}}$. As $\tilde{\mathcal{B}}$ is Hermitian, there are 3 real components (the diagonals) and 3 unique, complex off-diagonal components, for a total of 9 values (for each frequency, wavenumber, and Mach number). We transform the matrix into magnitude and phase, $\left|\tilde{\mathcal{B}}_{i, j}\right| e^{i \theta_{\tilde{\mathcal{B}}_{i . j}}}$, requiring a fit to 6 magnitudes and 3 angles for each frequency and Mach number combination.

In this transformation the magnitude of each matrix not only provides the overall intensity of occurrence of each resolvent mode, but also provides information on the strength of coupling between occurrences of one resolvent mode to another. The overall magnitude of $\tilde{\mathcal{B}}$ is shown in figure 14 across $S t=[0,1]$ for the six unique magnitude entries. An interesting observation is that from these 6 entries, they tend to cluster about three curves, $\tilde{\mathcal{B}}_{1,1}$ sits alone, while $\tilde{\mathcal{B}}_{2,1}$ and $\tilde{\mathcal{B}}_{2,2}$, and $\tilde{\mathcal{B}}_{3,1}, \tilde{\mathcal{B}}_{3,2}, \tilde{\mathcal{B}}_{3,3}$ cluster near each other respectively. Further, the general trends of these curves reflect energy curves computed through SPOD (details on SPOD can be found in ${ }^{16}$ ) using the same output domain, $r / D=[5,6]$ and $x / D=[0,30]$ of the fluctuating pressure, shown in figure 15. This comparison indicates that the covariance matrix seeks to redefine the resolvent basis into a new, more efficient basis similar to that of SPOD, the optimal basis for describing turbulent statistics. ${ }^{16}$

Returning to the definition of $\tilde{\mathcal{B}}, \tilde{\mathcal{B}}=\boldsymbol{\Sigma}_{z} \tilde{\boldsymbol{V}}^{*} \boldsymbol{W}_{f} \boldsymbol{S}_{f f} \boldsymbol{W}_{f} \tilde{\boldsymbol{V}} \boldsymbol{\Sigma}_{z}$, we can see that our current formulation includes the gains of the resolvent analysis, which may be accounted for directly. The gains of the first 


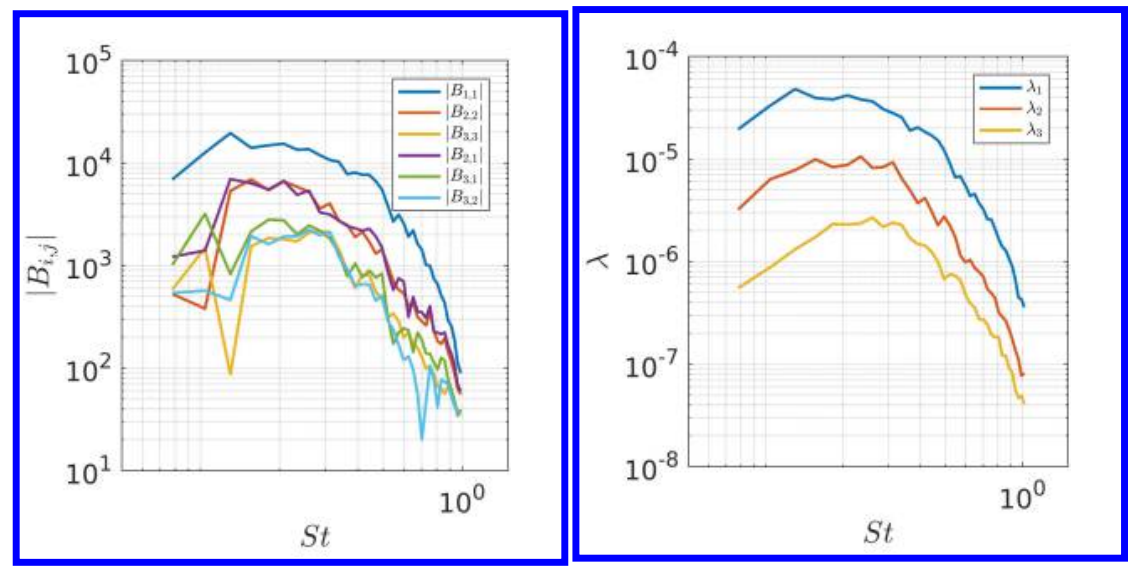

Figure 14. Magnitudes of the six unique entries of the $3 \times 3$

Figure 15. Three largest eigenvalues of the SPOD spectrum computed over the LES acoustic domain, $C_{z}$ for azimuthal wavenumber $m=0$. wavenumber, $m=0$.

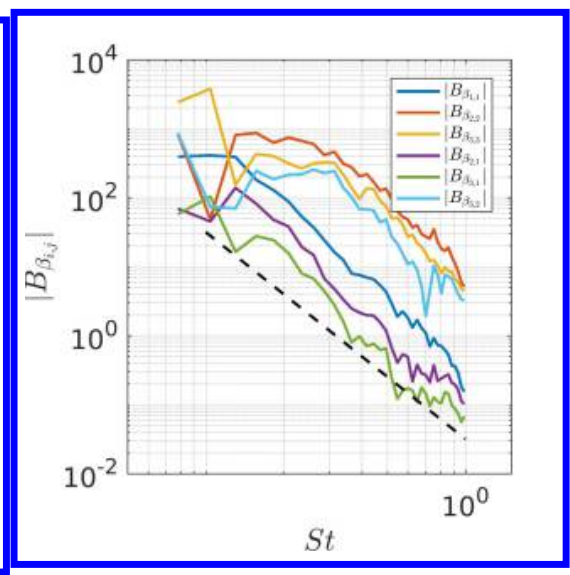

Figure 16. Resolvent gains for the first three modes across $S t=$ $[0,1]$ for azimuthal wavenumber $m=0$.

Figure 17. Magnitude of the forcing projection coefficients, $\boldsymbol{\beta}_{k} \boldsymbol{\beta}_{k}^{*}=\tilde{\boldsymbol{V}}^{*} \boldsymbol{W}_{f} \boldsymbol{f}_{k} \boldsymbol{f}_{k}^{*} \boldsymbol{W}_{f} \tilde{\boldsymbol{V}} . \quad$ The dashed line gives $\sim S t^{-3}$.

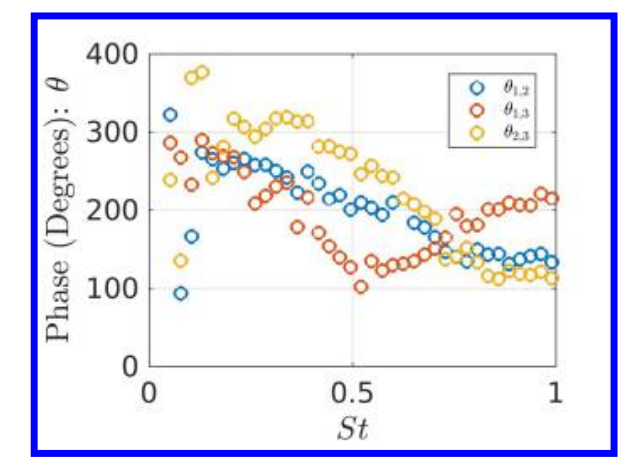

Figure 18. Phases of the three covariance matrix angles as a function of frequency.

3 resolvent modes are plotted in figure 16, with significant separation of the leading mode followed by two modes of similar gain. At first glance the gains do not appear to be entirely useful for removing any dependence of frequency on the magnitude of $\tilde{\mathcal{B}}$, however, removing the gains from the matrix, $\boldsymbol{\Sigma}_{z}^{-1} \tilde{\mathcal{B}} \Sigma_{z}^{-1}=$ 
$\tilde{\boldsymbol{V}}^{*} \boldsymbol{W}_{f} \boldsymbol{S}_{f f} \boldsymbol{W}_{f} \tilde{\boldsymbol{V}}$ gives figure 17. Here all of the entries have been transformed into curves of order -3 (the dashed line) with varying magnitudes, suggesting each entry may be modeled as, $\tilde{\mathcal{B}}_{i, j}(S t) \approx c_{i, j} S t^{-3}$, where $c_{i, j}$ represents a scalar amplitude. Although not presented here, similar trends (i.e. $\sim S t^{-3}$ ) are observed for azimuthal wavenumbers 1 and 2 , as well as for the $M_{j}=0.9$ jet. Figure 17 presents the mean of the ensemble of forcing projection coefficients, $\boldsymbol{\beta}_{k}=\tilde{\boldsymbol{V}}^{*} \boldsymbol{W}_{f} \boldsymbol{f}_{k}$, directly describing the intensity of each resolvent forcing mode found in the LES data. Interestingly, despite the first resolvent mode having the greatest amplification, the magnitude of the projection of the forcing into $\tilde{\boldsymbol{V}}$ is not as large, by many orders of magnitude, when compared to the magnitude of sub-optimal resolvent forcings.

Attention is now turned to the 3 phases of the covariance matrix with all three angles plotting in figure 18. It is difficult to suggest a general function to represent the phases. The phase curves, and to a similar degree the magnitude curves, seem to reflect the differences in dominant mechanisms with respect to frequency at $m=0$. Although there are no consistent patterns among the phases at low frequencies, $S t<0.25$, the phases do follow a linear trend from $S t=0.3-0.6$ and a second, but different, linear trend from $S t=0.6-1$.

\section{Summary and Outlook}

We leveraged the availability of a LES database to examine resolvent-mode-based reconstructions of the acoustic field of round, turbulent $M_{j}=1.5$ and $M_{j}=0.9$ jets. We represent the forcing cross-spectral density matrix with a truncated set of resolvent modes and approximate the amplitudes of the modes with best-fit expansion coefficients of realizations from the observed LES acoustic field. We find that relatively low-rank models consisting of as few as 3 resolvent modes can accurately reconstruct the acoustic field for the first two azimuthal modes for a $M_{j}=1.5$ jet, where as about 10 modes were required for the higher azimuthal mode $(m=2)$ and 10-20 modes were required for the $M_{j}=0.9$ jet. Based on the relative increase in radiation efficiency of the (low-rank) Kelvin-Helmholtz response of the jet, it is perhaps not surprising that the higher Mach number case can be captured at lower rank. In both cases, the use of an eddy-viscosity model in the resolvent formulation led to (vastly) superior results compared to a fixed Reynolds number. The results show that neglecting the off-diagonal components of the data CSD results in an underprediction by about $25 \mathrm{~dB}$, consistent with previous results using on linear models ${ }^{30,32}$ and demonstrated importance of "jitter" in wavepacket source models. ${ }^{23}$ However, neglecting the off-diagonal components of the estimated CSD instead, constructed via the resolvent basis, gave rather accurate reconstructions for $n=3$ and show that the resolvent basis is able to directly model correlations in the acoustic field.

Examination of the structure of the reduced-order covariance matrix showed dominance along the diagonal, explaining the success of reconstructions based solely upon the diagonal, and also provided signs of smooth, and generally simple, relations in terms of frequency dependence among all matrix components. Further work into the characteristics of reduced covariance matrices at other azimuthal wavenumbers and Mach numbers may produce general trends which may be leveraged for a jet-noise model. However, as $n$ increases, the parameter space which must be modeled increases by $n^{2}$. As such, this path forward for a transonic jet, where the inclusion of 20 modes is likely necessary for accurate predictions, may prove difficult unless additional assumptions, such as modeling only the diagonal term of $\tilde{\mathcal{B}}$, reducing the complexity to $n$, are taken.

Regardless of this difficulty, the ability of the resolvent basis to describe much of the acoustic field with 20 modes or less across multiple Mach numbers, a large range of frequencies, and the acoustically dominant azimuthal wavenumbers is promising. This indicates that the resolvent framework already contains the appropriate acoustic functions to describe jet noise, the next step must then be to generalize their correlations amongst each other such that they represent the effect of turbulence on noise generation.

\section{Acknowledgments}

The authors would like to thank André Cavalieri, Oliver Schmidt, and Georgios Rigas for many productive discussions on topics related to this paper. This research was supported by a grant from the Office of Naval Research (grant No. N00014- 16-1-2445) with Dr. Steven Martens as program manager. E.P. was supported by the Department of Defense (DoD) through the National Defense Science Engineering Graduate Fellowship (NDSEG) Program. The LES study was performed at Cascade Technologies, with support from ONR and NAVAIR SBIR project, under the supervision of Dr. John T. Spyropoulos. The main LES calculations were carried out on DoD HPC systems in ERDC DSRC. 


\section{References}

${ }^{1}$ McKeon, B. and Sharma, A., "A critical-layer framework for turbulent pipe flow," Journal of Fluid Mechanics, Vol. 658, 2010, pp. 336-382.

2 Jovanović, M. R. and Bamieh, B., "Componentwise energy amplification in channel flows," Journal of Fluid Mechanics, Vol. 534, 2005, pp. 145-183.

${ }^{3}$ Jeun, J., Nichols, J. W., and Jovanović, M. R., "Input-output analysis of high-speed axisymmetric isothermal jet noise," Physics of Fluids, Vol. 28, No. 4, 2016, pp. 047101.

${ }^{4}$ Schmidt, O. T., Towne, A., Rigas, G., Colonius, T., and Brès, G. A., "Spectral analysis of jet turbulence," Journal of Fluid Mechanics, Vol. 855, 2018, pp. 953-982.

$\rightarrow 5$ Lighthill, M. J., "On sound generated aerodynamically I. General theory," Proc. R. Soc. Lond. A, Vol. 211, No. 1107, 1952 , pp. $564-587$.

${ }^{6}$ Goldstein, M. E., "A generalized acoustic analogy," Journal of Fluid Mechanics, Vol. 488, 2003, pp. 315-333.

${ }^{7}$ Mollo-Christensen, E., "Jet noise and shear flow instability seen from an experimenters viewpoint," Journal of Applied Mechanics, Vol. 34, No. 1, 1967, pp. 1-7.

${ }^{8}$ Crow, S. C. and Champagne, F., "Orderly structure in jet turbulence," Journal of Fluid Mechanics, Vol. 48, No. 3, 1971, pp. $547-591$.

9 Jordan, P. and Colonius, T., "Wave packets and turbulent jet noise," Annual Review of Fluid Mechanics, Vol. 45, 2013, pp. 173-195.

${ }^{10}$ Papamoschou, D., "Wavepacket modeling of the jet noise source," International Journal of Aeroacoustics, Vol. 17, No. 1-2, 2018, pp. 52-69.

${ }^{11}$ Jeun, J. and Nichols, J. W., "Input-output analysis of Mach 0.9 jet noise," arXiv preprint arXiv:1806.09280, 2018.

$\checkmark{ }^{12}$ Crighton, D. G. and Gaster, M., "Stability of slowly diverging jet flow," Journal of Fluid Mechanics, Vol. 77, No. 02, 1976, pp. 397-413.

${ }^{13}$ Michalke, A., "Instability of a compressible circular free jet with consideration of the influence of the jet boundary layer thickness," NASA TM 75190, 1977.

${ }^{14}$ Cavalieri, A. V., Jordan, P., and Lesshafft, L., "Wave-packet models for jet dynamics and sound radiation," Applied Mechanics Reviews, Vol. 71, No. 2, 2019, pp. 020802.

$\checkmark{ }^{15}$ Lesshafft, L., Semeraro, O., Jaunet, V., Cavalieri, A. V., and Jordan, P., "Resolvent-based modeling of coherent wave packets in a turbulent jet," Physical Review Fluids, Vol. 4, No. 6, 2019, pp. 063901.

16 Towne, A., Schmidt, O. T., and Colonius, T., "Spectral proper orthogonal decomposition and its relationship to dynamic mode decomposition and resolvent analysis," Journal of Fluid Mechanics, Vol. 847, 2018, pp. 821-867.

${ }^{17}$ Brès, G. A., Jordan, P., Colonius, T., Le Rallic, M., Jaunet, V., and Lele, S. K., "Large eddy simulation of a Mach 0.9 turbulent jet," Proceedings of the Summer Program, Center for Turbulence Research, Stanford University, 2014

${ }^{18}$ Brès, G. A., Jaunet, V., Le Rallic, M., Jordan, P., Colonius, T., and Lele, S. K., "Large eddy simulation for jet noise: the importance of getting the boundary layer right," AIAA paper 2015-2535, 2015.

19 Towne, A., Lozano-Durán, A., and Yang, X., "Resolvent-based estimation of space-time flow statistics," Journal of Fluid Mechanics, Vol. 883, 2020.

${ }^{20}$ Zare, A., Jovanovi, M. R., and Georgiou, T. T., "Colour of turbulence," Journal of Fluid Mechanics, Vol. 812, 2017, pp. 636680 .

${ }^{21}$ Towne, A., Brès, G. A., and Lele, S. K., "A statistical jet-noise model based on the resolvent framework," AIAA Paper \#2017-3406, 2017.

${ }^{22}$ Pickering, E. M., Rigas, G., Sipp, D., Schmidt, O. T., and Colonius, T., "Eddy viscosity for resolvent-based jet noise models," 25th AIAA/CEAS Aeroacoustics Conference, 2019, p. 2454.

${ }^{23}$ Cavalieri, A. V., Jordan, P., Agarwal, A., and Gervais, Y., "Jittering wave-packet models for subsonic jet noise," Journal of Sound and Vibration, Vol. 330, No. 18-19, 2011, pp. 4474-4492.

${ }^{24}$ Brès, G. A., Ham, F. E., Nichols, J. W., and Lele, S. K., "Unstructured large-eddy simulations of supersonic jets," $A I A A$ Journal, 2017, pp. 1164-1184.

${ }^{25}$ Brès, G. A., Bose, S. T., Emory, M., Ham, F. E., Schmidt, O. T., Rigas, G., and Colonius, T., "Large eddy simulations of co-annular turbulent jet using a Voronoi-based mesh generation framework," AIAA paper 2018-3302, 2018.

$\checkmark{ }^{26}$ Brès, G. A., Jordan, P., Jaunet, V., Le Rallic, M., Cavalieri, A. V. G., Towne, A., Lele, S. K., Colonius, T., and Schmidt, O. T., "Importance of the nozzle-exit boundary-layer state in subsonic turbulent jets," J. Fluid Mech., Vol. 851, 2018, pp. $83-124$

${ }^{27}$ Chu, B.-T., "On the energy transfer to small disturbances in fluid flow (Part I)," Acta Mechanica, Vol. 1, No. 3, 1965, pp. 215-234.

${ }^{28}$ Pickering, E., Rigas, G., Nogueira, P. A., Cavalieri, A. V., Schmidt, O. T., and Colonius, T., "Lift-up, Kelvin-Helmholtz and Orr mechanisms in turbulent jets," arXiv preprint arXiv:1909.09737, 2019.

${ }^{29}$ Sinha, A., Rodríguez, D., Brès, G. A., and Colonius, T., "Wavepacket models for supersonic jet noise," Journal of Fluid Mechanics, Vol. 742, 2014, pp. 71-95.

- ${ }^{30}$ Cavalieri, A. V. and Agarwal, A., "Coherence decay and its impact on sound radiation by wavepackets," Journal of Fluid Mechanics, Vol. 748, 2014, pp. 399-415.

${ }^{31}$ Towne, A., Colonius, T., Jordan, P., Cavalieri, A. V., and Brès, G. A., "Stochastic and nonlinear forcing of wavepackets in a Mach 0.9 jet," 21st AIAA/CEAS Aeroacoustics Conference, 2015, p. 2217.

${ }^{32}$ Jordan, P., Colonius, T., Bres, G., Zhang, M., Towne, A., and Lele, S., "Modeling intermittent wavepackets and their radiated sound in a turbulent jet," Proceedings of the Summer Program, Center for Turbulence Research, Stanford University, 2014 , p. 241. 